\title{
Design and Development of a Multimodal Vest for Virtual Immersion and Guidance
}

\author{
Gonzalo García-Valle ${ }^{1(凶)}$, Manuel Ferre ${ }^{1}$, Jose Breñosa ${ }^{1}$, Rafael Aracil ${ }^{1}$, \\ Jose M. Sebastian ${ }^{1}$, and Christos Giachritsis ${ }^{2}$ \\ 1 Centre for Automation and Robotics (UPM-CSIC), \\ Universidad Politecnica de Madrid, Jose Gutierrez Abascal, 2, Madrid, Spain \\ \{gonzalo.gvalle,m.ferre, jose.brenosa, rafael.aracil, jose. sebastian\} @upm.es \\ 2 BMT Group Ltd., Goodrich House, 1 Waldegrave Road, Teddington, UK \\ cgiachritsis@bmtmail.com
}

\begin{abstract}
This paper is focused on the development of a haptic vest to enhance immersion and realism in virtual environments, through vibrotactile feedback. The first steps to achieve touch-based communication are presented in order to set an actuation method based on vibration motors. Resulting vibrotactile patterns helping users to move inside virtual reality (VR). The research investigates human torso resolution and perception of vibration patterns, evaluating different kind of actuators at different locations on the vest. Finally, determining an appropriate distribution of vibration patterns allowed the generation of sensations that, for instance, help to guide in a mixed or virtual reality environment.
\end{abstract}

Keywords: Haptic $\cdot$ Vest $\cdot$ Vibrotactile $\cdot$ Guidance $\cdot$ Virtual reality

\section{Introduction}

In the last decades, haptic technologies have been amply investigated as an ultimate way to obtain better results in human-machine interaction [1,2]. These technologies have a wide range of applications, from industry to training and entertainment [3]. Another relatively new technology with a broad range of applications is virtual, mixed or augmented reality [4]. A combination of both previous technologies can generate systems in which haptic technology produces a significant improvement in immersion and realism that a user experiences when inside of a virtual system $[5,6]$.

Here, the development of a haptic vest for counter terrorist police training within a mixed reality environment is reported.

The proposed haptic vest seeks to improve immersion and realism of interaction in virtual environments, through several vibrotactile stimuli such as impact effects, thermal effects or touch-based communication between members of the same training team. In this paper, we present the first steps to achieve touchbased communication based on vibration motors allowing the creation of vibrotactile patterns that help users to move inside virtual environments.

(C) Springer International Publishing Switzerland 2016

F. Bello et al. (Eds.): EuroHaptics 2016, Part II, LNCS 9775, pp. 251-262, 2016.

DOI: $10.1007 / 978-3-319-42324-1 \_25$ 


\section{$1.1 \quad$ Related Works}

Over the past two decades, research in haptic interfaces and VR has been constantly evolved. There are several examples about research that try to join both concepts, creating new displays to provide haptic feedback in virtual environments $[7,8]$. Previous haptic vests, like Tactavest $[9,10]$, a tactile vest for astronauts [11] or the vest developed by Jones et al. [12], that use different actuation methods distributed at various trunk areas. These vests were designed for different applications (including military coordination [13], emotional therapy [14] and immersion in VR systems). Other methods have used vibration motors capable of generating complex sensations through haptic illusions [15].

Actuators distribution on the vest required a previous study about two-point vibration discrimination distance for creating sophisticated patterns, but there are no studies about that in selected areas. Moreover, even though there is a study on discrimination of vibration patterns at the back, there are no data on discrimination of vibration patterns at shoulders [16].

Vibrotactile vests can be used for navigation in VR as well as real world environments (e.g., for the navigation of the blind $[17,18]$ ). There are several researchers that use a vest, or other wearable interfaces, like a belt [19] for guiding a user in a specific path, placing vibration motors on upper back instead of the shoulders and upper trunk [20]. Dharma et al. [21] have applied the same procedure with actuators in medium and lower back and abdomen. Finally, Prasad et al. have created a haptic vest for obstacle avoidance [22] and guidance of bikers [23].

\subsection{Research Objectives}

The main objective is the development of a vest capable of delivering several haptic feedback stimuli, allowing to improve realism and immersion in virtual environments. The vest design includes actuators generating vibrotactile stimulation at different torso locations.

Distribution of actuators in torso is organized as follows: tactile actuators are placed on shoulders, upper back and upper chest, since these areas are commonly used to conduct touch-based communication among counter terrorist officers during operation. Thermal effects are placed on lower back and abdomen, and other haptic effects are located throughout entire torso, corresponding actuators are positioned between thermal and vibration actuators.

Two main tests were carried out:

- A test to determine the minimum distance (discrimination threshold) between two vibration stimuli on the chosen areas that is necessary in order to be perceived as distinct stimuli.

- A preliminary evaluation to determine if the vest can be used like a method to assist navigation into a VR system. 


\section{Haptic Actuation}

Two different actuation methods were considered to generate tactile stimuli: Electrical Muscle Stimulation (EMS) and vibration motors. However, after making an analysis about both methods, it was decided to use vibration motors due to induced sensations being more reliable and comfortable for users.
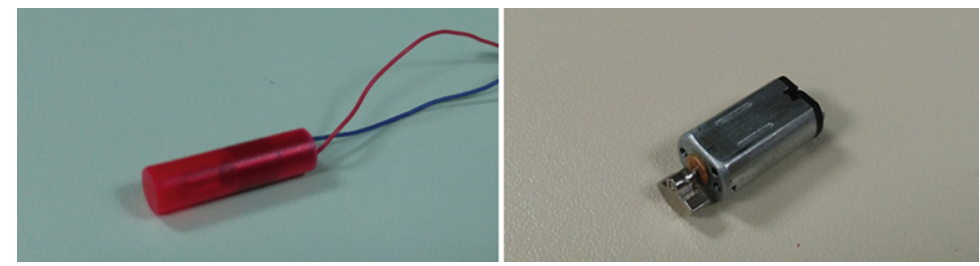

Fig. 1. Motor "304-116" (left); motor "308-102" (right)

Following several tests with ten different motors (including Linear Resonance Actuators (LRA)) two motors were selected for further development and testing: "304-116" model and "308-102" model, both from Precision Microdrives Ltd (Fig. 1). These two motors can produce high frequencies allowing easy generation of haptic sensations. Moreover, LRA were ruled out because of low vibration intensity that is not easily appreciable on stimulated areas. The technical characteristics of the two motors are shown in Table 1.

Table 1. Characteristics of the vibration motors

\begin{tabular}{l|c|l}
\hline Characteristics & “304-116” motor & "308-102” motor \\
\hline Rated voltage $(\mathrm{V})$ & 3 & 4.5 \\
\hline Rated current $(\mathrm{mA})$ & 44 & 145 \\
\hline Rated speed $(\mathrm{rpm})$ & 14000 & 19000 \\
\hline Rated frequency $(\mathrm{Hz})$ & 255 & 330 \\
\hline
\end{tabular}

Motor "308-102" has greater rated voltage and current (4.5 V and $145 \mathrm{~mA}$ ) than motor "304-116" (3 V and $44 \mathrm{~mA})$. However, these differences are not an issue since it implies only minor changes in control circuit. Moreover, motors work with different rated frequency $(255 \mathrm{~Hz}$ for " $304-116$ " motor and $330 \mathrm{~Hz}$ for "308-102" motor), that are easily detected by human skin, since its vibration sensitivity range is between 30 and $500 \mathrm{~Hz}$, approximately.

Actuators are controlled by a LilyPad Arduino with several Pulse-Width Modulation (PWM) outputs that can be used for varying frequency motors. 


\section{Resolution Experiment}

The following experiment aims to find out the two-point discrimination threshold on shoulders, upper torso and upper back. The obtained results are used to determine tactile actuators distribution on those areas.

\subsection{Stimuli Patterns}

The areas selected to position the two actuators were divided into seven small sections and the discrimination threshold was obtained for each actuator. Two different tests were carried out.

The first test was performed with "304-116" motor, using a row of ten motors separated from each other $10 \mathrm{~mm}$. The mesh was placed in seven torso locations and the actuators were programmed to reproduce a sequence of 15 vibrations (1-second vibration followed by 3 -second break off when subjects have to tell how many vibration sources are working in previous vibration). Figure 2 shows the row with "304-116" motors and how it is attached on a participant during the tests.

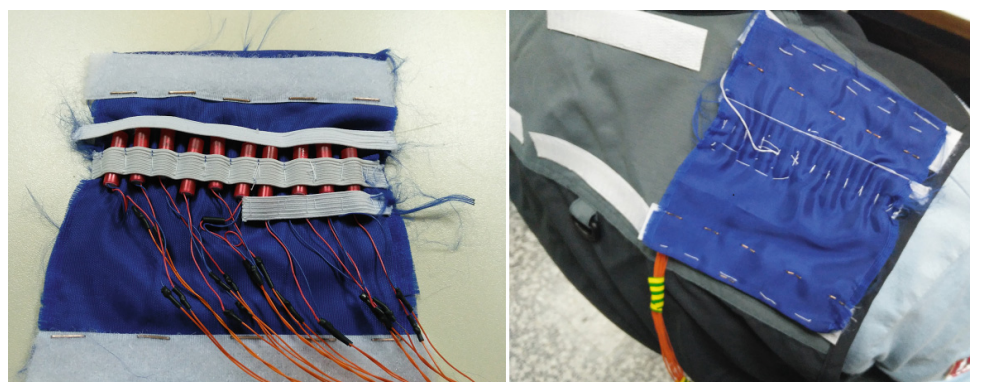

Fig. 2. 304-116 motors row (left); user during tests (right)

Two different motors vibrate in each phase and participants indicate if one or two motors are vibrating. Vibrations appear in random order while several repetitions are produced, avoiding the sequence is easily identifiable. In addition, vibrations are produced at all possible distances $(10,20,30, \ldots 90 \mathrm{~mm})$, establishing discrimination distance for each participant and each area. Subsequently, the same procedure is done with "308-102" motors. However, since motors are bigger only 6 could be placed at a distance of $20 \mathrm{~mm}$ from each other. Sequence is reproduced during the test in just four areas to compare likely perception differences between different motors.

At the beginning of the first phase, participants experience demo vibrations in order to ensure that sensations can be easily appreciated. With those vibrations, subjects can do the entire test and express their sensations easily and without confusion. 


\subsection{Participants}

Twenty four participants took part in the test (15 males and 9 females) with age from 22 to 35 years old. One of them indicated a neuropathology that affects the right side sensibility, consequently, the results of that subject were excluded from further analysis. 20 participants were students of Universidad Politecnica de Madrid and 4 participants were external from the institution.

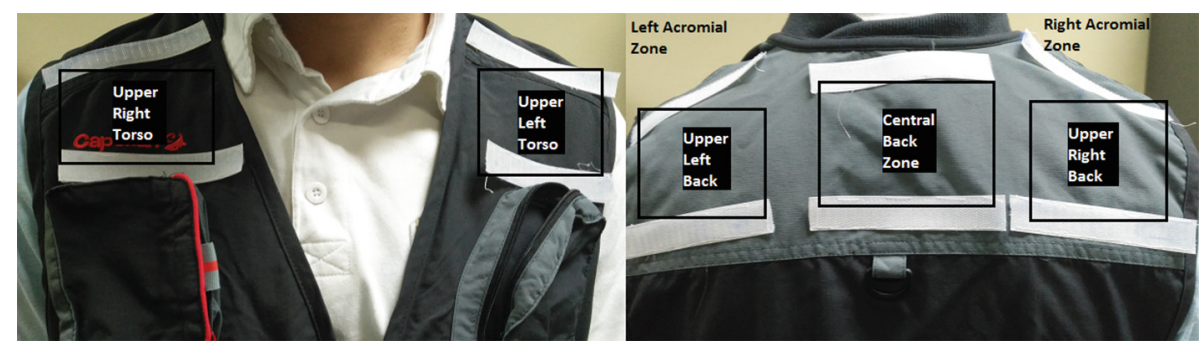

Fig. 3. Analysed areas during first experiment

\subsection{Results}

Data from individual performances were analysed to obtain the discrimination threshold. The resolution value is a number between 10 and $90 \mathrm{~mm}$, since there were the minimum and maximum distances where two motors have been placed. The results can only take ten-by-ten values $(10,20,30 \ldots, 90)$.

Table 2. Median values for each area $(\mathrm{mm})$

\begin{tabular}{l|l|l}
\hline Areas & “304-116" motor & "308-102” motor \\
\hline Upper Right Torso (U.R.T) & 60 & 60 \\
\hline Right Acromial Zone (R.A.Z) & 50 & - \\
\hline Upper Right Back (U.R.B) & 50 & 60 \\
\hline Central Back Zone (C.B.Z) & 50 & - \\
\hline Upper Left Torso (U.L.T) & 40 & 40 \\
\hline Left Acromial Zone (L.A.Z) & 40 & - \\
\hline Upper Left Back (U.L.B) & 50 & 40 \\
\hline
\end{tabular}

Once the experimental data for each participant are collected, the median discrimination value was obtained. Due to high standard deviation, the median value was selected to represent discrimination performance, in order to facilitate the rejection of outliers through a statistical analysis. The torso areas for which 
discrimination values were obtained are shown in Fig. 3. The medians discrimination values for each torso area are shown in Table 2.

Finally, results can be divided into different population groups (males and females, "304-116" motor and "308-112" motor, etc.), verifying the perception differences between them. The discrimination values between male and female participants are shown in Table 3.

Table 3. Comparison of perception between male and female

\begin{tabular}{l|l|l|l|l|l}
\hline Areas & Male median $(\mathrm{mm})$ & Male SD & Female median $(\mathrm{mm})$ & Female SD & p-value \\
\hline U.R.T & 65 & 26.44 & 60 & 23.98 & 0.513 \\
\hline R.A.Z & 60 & 23.45 & 30 & 20.88 & 0.1004 \\
\hline U.R.B & 60 & 17.06 & 25 & 5.48 & 0.0074 \\
\hline C.B.Z & 50 & 19.32 & 60 & 23.98 & 0.6054 \\
\hline U.L.T & 60 & 23.32 & 40 & 21.67 & 0.1058 \\
\hline L.A.Z & 30 & 30.95 & 60 & 19.36 & 0.264 \\
\hline U.L.B & 50 & 25.55 & 40 & 22 & 0.378 \\
\hline
\end{tabular}

\section{Guidance Experiment}

A preliminary evaluation is performed to determine if the haptic vest can be used for navigation within a training VR environment (e.g., when environmental conditions - such as presence of smoke - result in visual impairment). The test allows knowing if patterns generated for driving users and validating actuators distribution. Finally, it allows knowing if a sensory system is needed to adjust the guidance to path requirements.

\subsection{Stimuli Patterns}

First, vibration motors are distributed on established areas: shoulders, upper torso and back. Twelve motors are placed (6 of each type previously selected) on each shoulder at $30 \mathrm{~mm}$ away from each other. Placing motors closer than discrimination distance $(30 \mathrm{~mm})$, allows perception of a widespread feeling of vibration.

The objective was to guide the users through a path created by vibrotactile patterns representing turns, validating actuators placement and created patterns. Participants were asked to follow a path consisting on five turns $\left(90^{\circ}\right.$ angles). The duration of the patterns were randomly selected, analysing turn amplitude later. Due to random duration, users probably do not turn $90^{\circ}$ (it is only a way of representing it).

Patterns are as follows: first, "row 1" vibrates (closer to neck), later "row 2" vibrates (intermediate) and, finally, "row 3" vibrates (external row); creating a 
directional sensation that drives users towards a direction. Vibrations on right shoulder have lower intensity than vibrations on left shoulder to analyse how vibration intensity affects user's comfort. The distribution of motors across the rows can be seen in Fig.4. A participant during the experiment can be seen in Fig. 5.

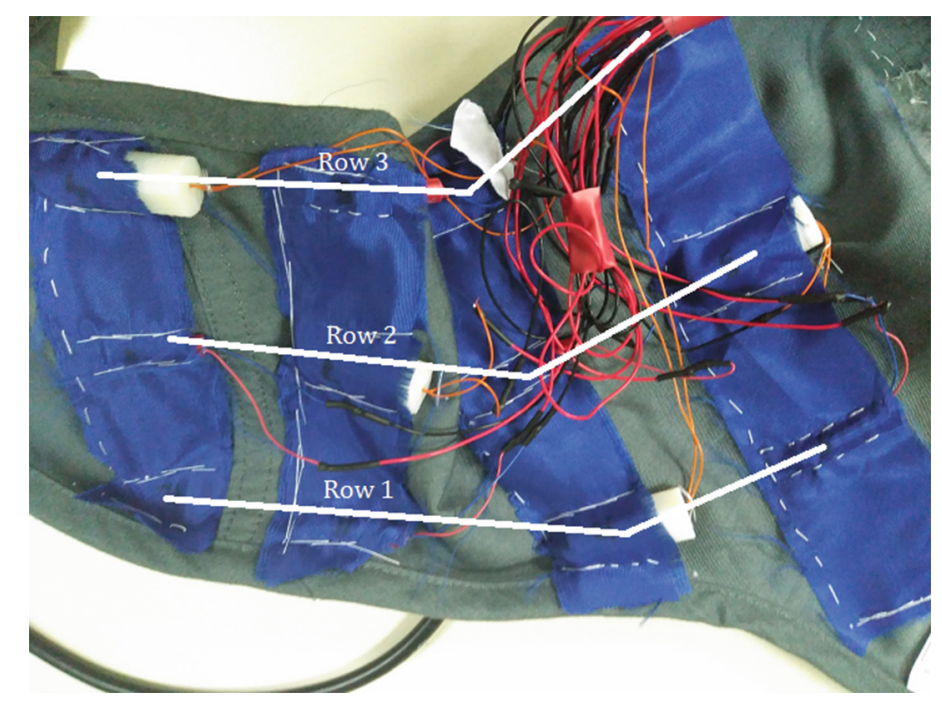

Fig. 4. Motor distribution in rows

The test included two types of stimuli:

- First: each row vibrates for 100 msec sequentially for fifteen times resulting in a total vibration duration of $4.5 \mathrm{~s}$.

- Second: each row vibrates for $300 \mathrm{msec}$ sequentially for six times resulting in a total vibration duration of $5.4 \mathrm{~s}$.

\subsection{Participants}

Five participants took part in the test (4 males and 1 female), with ages between 22 and 28. None of them indicate a neuropathology that affect their sensibility. All participants are students of Universidad Politecnica de Madrid (UPM).

\subsection{Results}

Data analysis was performed to find out the correct follow-up of turns during guidance, user comfort and ability to reach the end of the original path. Analysis of users' turns during path following showed that users correctly 


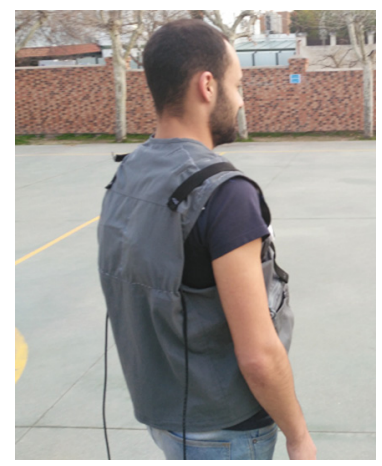

Fig. 5. Participant during guidance test

(100\% success rate) perceived the direction of rotation suggested by the stimuli. However, the turning angle between users were variable during a same vibration. Table 4 shows the results of the turning angles for the shorter and longer stimuli (shorter phase/larger phase).

Table 4. Turn angles during the path $\left(^{\circ}\right)$

\begin{tabular}{l|l|l|l|l|l}
\hline Subjects & 1st turn & 2nd turn & 3rd turn & 4th turn & 5 th turn \\
\hline 1 & $120 / 150$ & $120 / 135$ & $120 / 145$ & $170 / 135$ & $120 / 150$ \\
\hline 2 & $120 / 150$ & $120 / 150$ & $120 / 170$ & $120 / 160$ & $170 / 170$ \\
\hline 3 & $120 / 160$ & $145 / 210$ & $90 / 100$ & $90 / 120$ & $160 / 150$ \\
\hline 4 & $135 / 150$ & $170 / 180$ & $170 / 150$ & $170 / 180$ & $145 / 180$ \\
\hline 5 & $80 / 160$ & $70 / 170$ & $100 / 160$ & $150 / 150$ & $150 / 150$ \\
\hline
\end{tabular}

Moreover, even though there were differences between right and left turns due to the intensity of the stimuli, it seems that participants felt comfortable with the vibrotactile stimuli.

Figure 6 shows the path followed by three subjects for the two different vibration times. Although the path is completed correctly, shorter vibrations resulted in more reliable path executions. Original path is also shown in Fig. 6.

Finally, only three participants appreciate directionality sensation during turning when vibrations have a duration of $300 \mathrm{~ms}$, therefore it is necessary to improve the patterns to achieve a more intuitive drivability.

\section{Discussion}

Analysis of median values for distance (two-point) discrimination in different torso areas showed that the left side is more sensitive to vibrotactile stimulation. 

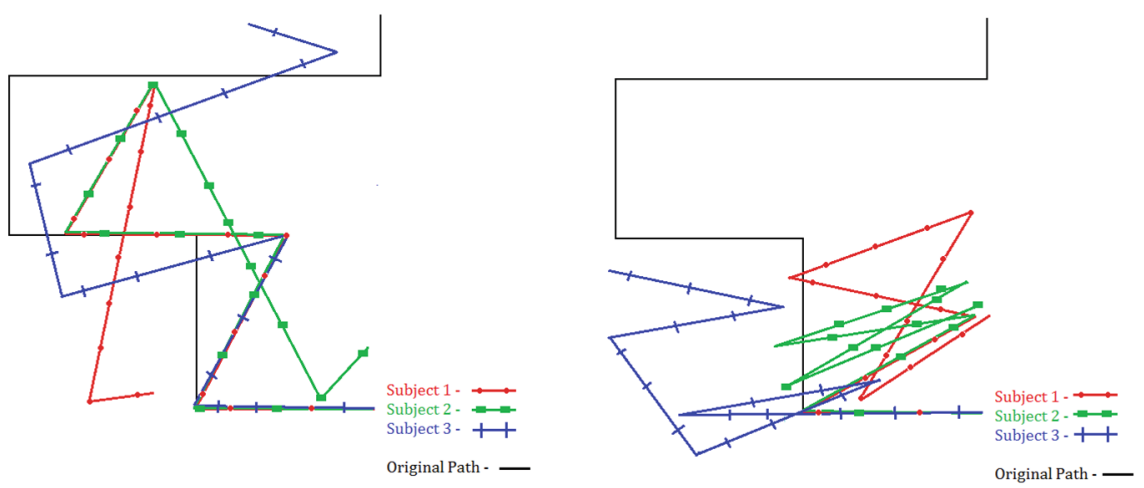

Fig. 6. Path followed by 3 subject with $100 \mathrm{~ms}$ vibrations (left); path followed by 3 subject with $300 \mathrm{~ms}$ vibrations (right)

Those differences may be due to distinct sensibility between body sides (left and right), although that scenarios must be confirmed in the future.

Figure 7 shows the discrimination values obtained for the two different motors: the left one ("304-116") and the right one ("308-102"), obtaining a maximum value of $60 \mathrm{~mm}$. Since the actuators distribution on vest is done according to the maximum resolution value to ensure patterns perception since motors are placed at a distance of $70 \mathrm{~mm}$. Moreover, additional motors are interspersed at $35 \mathrm{~mm}$, allowing the creation of a generalized vibration on stimulated areas.

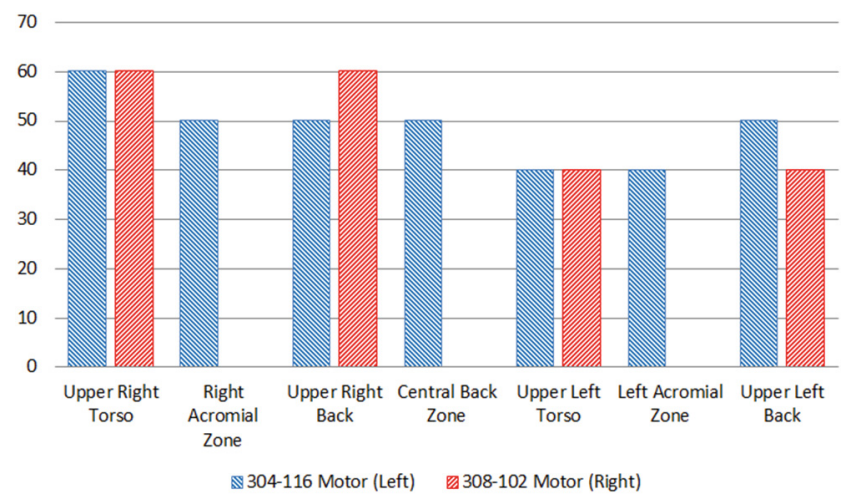

Fig. 7. Discrimination distances obtained during first experiment

Statistical analysis showed that there was no difference in two-point discrimination at different torso locations (shoulders, upper torso and upper back) ( $\mathrm{p}$-values $>0.05)$. Moreover, it seems that two-point discrimination is independent of gender and type of motor used to generate the stimuli. There are few 
significant results (five in all with p-value $<0.05$ ) that can be taken as random values and do not means that perception is similar between population groups.

Additional evaluation test were carried out to investigate the usefulness of the haptic vest in guiding users within a VR environment. For this purpose, different vibration patterns with varied intensity and duration were generated represent turning directions, enabling users do turns correctly in a designed path.

Results showed that participants did not feel discomfort when perceiving the stimuli during navigation. In addition, it was found that lower intensity produced better navigation results even though users turned effectively with both intensities. The choice of right side for lower intensity is random, since comfortability is not related with greater or lower discrimination distance.

Moreover, while all sequences have the same duration, turn angles depend on the participant. Furthermore, turning patterns represented by shorter stimuli resulted in more reliable path execution, since turns are smaller than turns done with larger stimuli. Therefore, vibration time is an important parameter to consider on the design because larger vibrations produce sharp turns since subjects continue turning until the end of vibrations.

To achieve a required angle, it is proposed the integration of a sensory system (gyro and accelerometer), that indicates when the user has done a correct turn to continue the path and, at that moment, stop vibrations or stimulate the user for starting to walk towards a specific point.

Finally, some users commented they did not feel that the vibrational patterns conveyed any directions. Therefore, it may be necessary to generate more elaborate patterns conveying clearer directional instructions. It is observed that perceived sensations are similar to feelings noticed by people guidance into a real environment through touch-based communication.

\section{Conclusions and Future Work}

This paper proposed the development of a haptic vest to increase the immersion and realism in a VR. In order to achieve this, several haptic feedback types have been analysed and tactile feedback has been implemented. The first step was to include tactile actuators on shoulders, upper torso and upper back.

The two-point vibration discrimination distance for chosen areas was determined, in order to understand how actuators have to be placed on the vest to generate distinct vibration patterns. Sensation has been suggested to induce movement that will vary based on the specific pattern created.

In addition, the vest was evaluated as an interface to assist users guidance into a VR environment. Results showed that participant could make use of the vibration patterns to navigate. Further improvements could be possible through the incorporation of additional sensory systems (e.g., accelerometer and gyroscopes) or the development of more elaborate vibration patterns. The vest with all actuators (vibrotactile, thermal) will be integrated in a VR system to improve immersion and realism. 


\section{References}

1. Silva, A.J., Ramirez, O.A., Vega, V.P., Oliver, J.P.O.: Phantom omni haptic device: kinematic and manipulability. In: Electronics, Robotics and Automotive Mechanics Conference (CERMA 2009), pp. 193-198. IEEE (2009)

2. Kuschel, M., Di Luca, M., Buss, M., Klatzky, R.L.: Combination, integration in the perception of visual-haptic compliance information. IEEE Trans. Haptics 3(4), 234-244 (2010)

3. Schmidt, H.: HapticWalker-a novel haptic device for walking simulation. In: Proceedings of EuroHaptics (2004)

4. Azuma, R., Baillot, Y., Behringer, R., Feiner, S., Julier, S., MacIntyre, B.: Recent advances in augmented reality. IEEE Comput. Graph. Appl. 21(6), 34-47 (2001)

5. Bujanda, I.G.: Design and control of multi-finger haptic devices for dexterous manipulation. Ph.d. thesis, Industriales (2013)

6. Hecht, D., Reiner, M.: Sensory dominance in combinations of audio, visual and haptic stimuli. Exp. Brain Res. 193(2), 307-314 (2009)

7. Bau, O., Poupyrev, I.: Revel: tactile feedback technology for augmented reality. ACM Trans. Graph. (TOG) 31(4), 1-11 (2012)

8. Bau, O., Poupyrev, I., Israr, A., Harrison, C.: TeslaTouch: electrovibration for touch surfaces. In: Proceedings of the 23nd Annual ACM Symposium on User Interface Software and Technology, pp. 283-292. ACM (2010)

9. Lindeman, R.W., Page, R., Yanagida, Y., Sibert, J.L.: Towards full-body haptic feedback: the design and deployment of a spatialized vibrotactile feedback system. In: Proceedings of the ACM Symposium on Virtual Reality Software and Technology, pp. 146-149. ACM (2004)

10. Lindeman, R.W., Sibert, J.L., Lathan, C.E., Vice J.M.: The design and deployment of a wearable vibrotactile feedback system. In: Eighth International Symposium on Wearable Computers (ISWC 2004), vol. 1, pp. 56-59. IEEE (2004)

11. Van Erp, J.B.F., Van Veen, H.A.H.C.: A multi-purpose tactile vest for astronauts in the international space station. In: Proceedings of Eurohaptics, pp. 405-408, Dublin, Ireland. ACM Press (2003)

12. Jones, L.A., Nakamura, M., Lockyer, B.: Development of a tactile vest. In: 12 th International Symposium on Haptic Interfaces for Virtual Environment and Teleoperator Systems (HAPTICS 2004), Proceedings, pp. 82-89. IEEE (2004)

13. Cummings, D., Lucchese, G., Prasad, M., Aikens, C., Ho, J., Hammond, T.: Haptic and AR interface for paratrooper coordination. In: Proceedings of the 13th International Conference of the NZ Chapter of the ACM's Special Interest Group on Human-Computer Interaction, pp. 52-55. ACM (2012)

14. Bonanni, L., Vaucelle, C., Lieberman, J., Zuckerman, O.: Taptap: a haptic wearable for asynchronous distributed touch therapy. In: Extended Abstracts on Human Factors in Computing Systems (CHI 2006), pp. 580-585. ACM (2006)

15. Israr, A., Poupyrev, I.: Tactile brush: drawing on skin with a tactile grid display. In: Proceedings of the SIGCHI Conference on Human Factors in Computing Systems, pp. 2019-2028. ACM (2011)

16. Eskildsen, P., Morris, A., Collins, C.C., Bach-y Rita, P.: Simultaneous and successive cutaneous two-point thresholds for vibration. Psychon. Sci. 14(4), 146-147 (1969)

17. Ertan, S., Lee, C., Willets, A., Tan, H., Pentland, A.: A wearable haptic navigation guidance system. In: Second International Symposium on Wearable Computers, Digest of Papers, pp. 164-165. IEEE (1998) 
18. Colwell, C., Petrie, H., Kornbrot, D., Hardwick, A., Furner, S.: Haptic virtual reality for blind computer users. In: Proceedings of the Third International ACM Conference on Assistive Technologies, pp. 92-99. ACM (1998)

19. Van Erp, J.B.F., Van Veen, H.A.H.C., Jansen, C., Dobbins, T.: Waypoint navigation with a vibrotactile waist belt. ACM Trans. Appl. Percept. (TAP) 2(2), 106-117 (2005)

20. Jones, A.L., Lockyer, B., Piateski, E.: Tactile display and vibrotactile pattern recognition on the torso. Adv. Robot. 20(12), 1359-1374 (2006)

21. Dharma, A.A.G., Oami, T., Obata, Y., Yan, L., Tomimatsu, K.: Design of a wearable haptic vest as a supportive tool for navigation. In: Kurosu, M. (ed.) HCII/HCI 2013, Part IV. LNCS, vol. 8007, pp. 568-577. Springer, Heidelberg (2013)

22. Prasad, M., Taele, P., Olubeko, A., Hammond, T.: HaptiGo: a navigational tap on the shoulder. In: Haptics Symposium (HAPTICS 2014), pp. 339-345. IEEE (2014)

23. Prasad, M., Taele, P., Goldberg, D., Hammond, T.A.: Haptimoto: turn-by-turn haptic route guidance interface for motorcyclists. In: Proceedings of the SIGCHI Conference on Human Factors in Computing Systems, pp. 3597-3606. ACM (2014) 